\title{
Simulation of Plasma Flows in Divergent Magnetic Nozzles
}

\author{
Mario Merino and Eduardo Ahedo
}

\begin{abstract}
Images from the simulation code DIMAGNO illustrate the roles of pressure, electric, and magnetic forces in the 2-D plasma expansion in a magnetic nozzle and the generation of thrust.
\end{abstract}

Index Terms-Magnetic nozzles, multi-fluid plasmas, plasma simulation, plasma thrusters.

$\mathbf{M}$ AGNETIC NOZZLES, created by a longitudinal magnetic field, are being proposed as plasma acceleration devices in advanced propulsion systems. There is a clear analogy between the subsonic-to-supersonic flows of plasmas in a magnetic nozzle and of neutral gases in a solid nozzle. However, plasma dynamics in a magnetic nozzle are more complex because of the existence of electric currents, different acceleration mechanisms, and downstream detachment. These plasma features can be analyzed with the code DIMAGNO, which implements a 2-D steady-state model of the plasma expansion in a divergent magnetic nozzle [1]. Typically, the plasma is collisionless, fully ionized, current free, low beta (i.e. low density), and supersonic. The strength of the guide magnetic field assures that electrons are strongly magnetized, whereas ions are partially magnetized. The use of the method of characteristic lines (i.e., ion streamlines and Mach lines) for integrating the hyperbolic equations makes the code fast, accurate, and efficient.

Fig. 1 shows a 2-D map of plasma density and several pairs of electron and ion stream tubes with the same cross section at the nozzle throat. There, the plasma jet is sonic, with radially nonuniform density, and no ion rotation, which are typical characteristics of, at least, a helicon-source plasma. The electron and magnetic streamtubes coincide but, due to weak magnetization, ion streamtubes are less divergent, except at the plasma-vacuum edge, where quasineutrality causes the ion and electron tubes at the edge to match. This matching results from an increased electric field perpendicular to $\boldsymbol{B}$, which also fosters plasma rarefaction at the edge. The relative focusing of the plasma jet compensates partially the nozzle divergence. The separation of ion and electron streamtubes breaks the longitudinal current-density ambipolarity (here imposed at the throat) while quasineutrality and the global current-free condition are still satisfied.

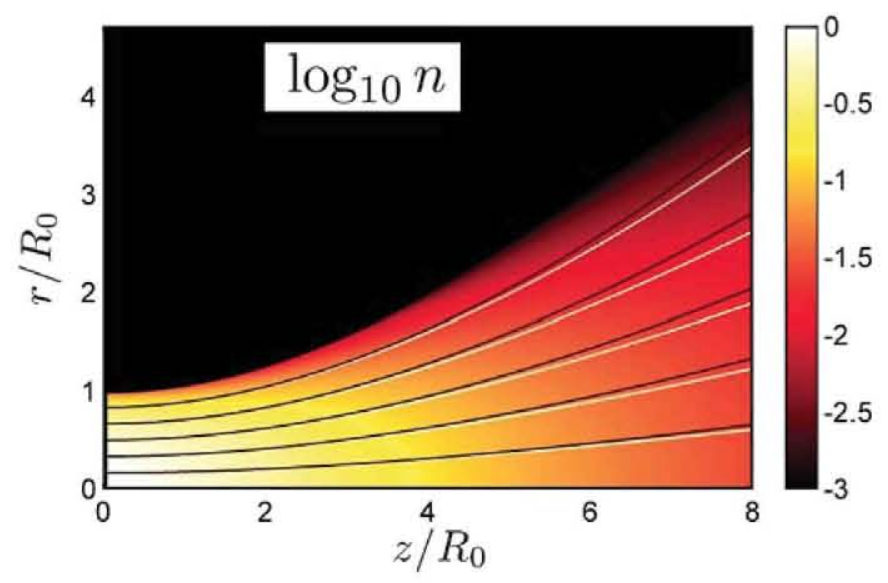

Fig. 1. Logarithmic map of the normalized plasma density and pairs of (dark) electron and (light) ion streamtubes.

Plasma confinement and (supersonic) acceleration are governed by the ion and electron momentum equations

$$
m_{i} n \vec{u}_{i} \cdot \nabla \vec{u}_{i}-\vec{j}_{i} \times \vec{B}=e n \vec{E}=-T_{e} \nabla n+\vec{j}_{e} \times \vec{B}
$$

where symbols are conventional. In general, $j_{i} \ll j_{e} \approx j_{\theta e}$ and ions are driven almost exclusively by the ambipolar electric force. The electron equation states the balance among the pressure, electric, and magnetic forces. Fig. 2(a) shows the relative weight of the three radial forces, the expanding pressure being compensated by both electric and magnetic confinement. The radial electric force, negligible at the nozzle throat, develops downstream and compels the ions to diverge. Fig. 2(b) shows the more complex axial balance. The axial pressure force accelerates near the axis and decelerates near the edge. Thus, near the beam center, $f_{z, p} \approx f_{z, e}$, whereas, near the edge, $f_{z, m} \approx-f_{z, p}$. This behavior, unrecoverable by a 1-D model, is caused by the strong 2-D density rarefaction.

The purpose of a propulsive magnetic nozzle (PMN) is to increment the thrust imparted by the plasma jet. In a solid nozzle, this is achieved by the gas pressure on the walls. In a PMN, thrust comes from the reaction force to $f_{z, m}=-j_{\theta e} B_{r}$ on the thruster magnetic circuit. Fig. 2(c) shows this force (and its integral along a $z=$ const. section, $F_{z, m}$ ) showing that it is concentrated near the edge on the near plume. The thrust gain due to the PMN is the integral of $F_{z, m}$. A positive gain requires the Hall current $j_{\theta e}$ to be diamagnetic, which, fortunately, is the one naturally occurring in PMN and also the condition supporting a radially confining and axially accelerating magnetic force. Furthermore, a diamagnetic Hall current induces a magnetic field that opposes the applied field and increases the divergence of the resulting nozzle and plasma jet, a scenario contrary to the 

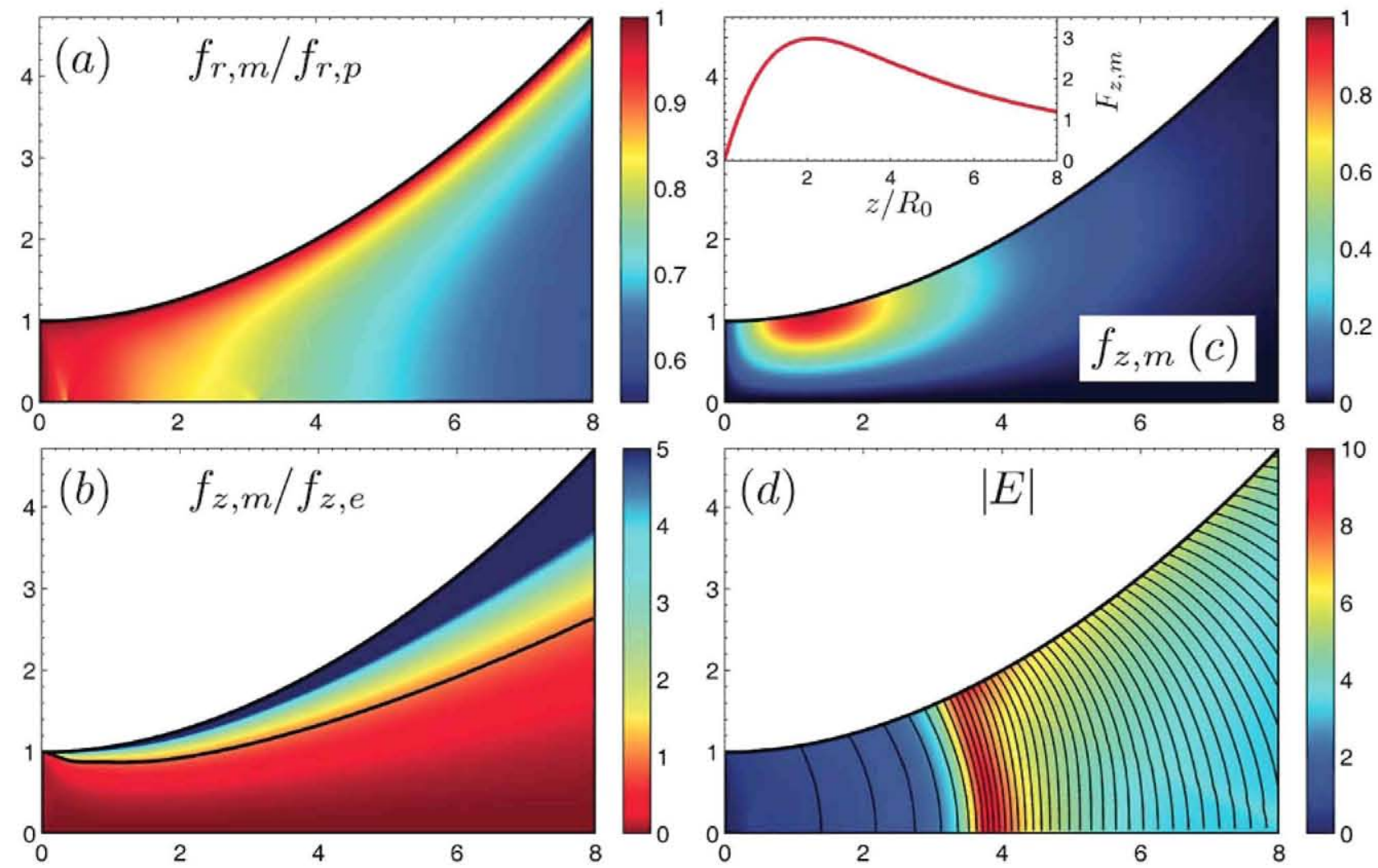

Fig. 2. (a) Magnetic-to-pressure radial force ratio from the radial balance of electron forces $f_{r, p}=f_{r, e}+f_{r, m}$. (b) Magnetic-to-electric radial force ratio from the axial balance of electron forces $f_{z, e}=f_{z, p}+f_{z, m}$; the black line separates regions of positive and negative pressure axial force. (c) Magnetic axial force per unit-volume, $f_{z, m}$, and its integral, $F_{z, m}$, on a $z=$ const disk. (d) Ambipolar electric field and equipotential lines for plasmas with a 0.2 fraction of nine times hotter electrons at the nozzle throat. Axes are the same than in Fig. 1.

magnetic-stretching detachment theory (see [2] and references therein).

Fig. 2(d) shows that DIMAGNO can also simulate the expansion of plasmas with a small extra population of hot electrons. These plasmas would explain the formation of currentfree double layers in helicon-source plasma beams (see [3] and references therein). In Fig. 2(d), the red curved region of maximum electric field is the steepened layer, with an electric potential fall on the order of the energy of hot electrons. That layer acts as a formidable barrier for "cold" electrons, so that the initially small population of hot electrons dominates completely the plasma flow downstream of the layer.
In conclusion, the code DIMAGNO has produced images that provide much understanding of the multifaceted plasma behavior in a magnetic nozzle.

\section{REFERENCES}

[1] E. Ahedo and M. Merino, "Two-dimensional supersonic plasma acceleration in a magnetic nozzle," Phys. Plasmas, vol. 17, no. 7, p. 073 501, Jul. 2010.

[2] E. Ahedo and M. Merino, "On plasma detachment in propulsive magnetic nozzles," Phys. Plasmas, vol. 18, no. 5, p. 053 504, May 2011.

[3] E. Ahedo, "Double-layer formation and propulsive assessment for a threespecies plasma expanding in a magnetic nozzle," Phys. Plasmas, vol. 18, no. 3, p. 033510 , Mar. 2011. 